\title{
ON BLOCK IDEMPOTENTS OF MODULAR GROUP RINGS
}

\author{
MASARU OSIMA
}

To the memory of TADASI NAKayama

We consider a group $G$ of finite order $g=p^{a} g^{\prime}$, where $p$ is a prime number and $\left(p, g^{\prime}\right)=1$. Let $\Omega$ be the algebraic number field which contains the $g$-th roots of unity. Let $K_{1}, K_{2}, \ldots, K_{n}$ be the classes of conjugate elements in $G$ and the first $m(\leqq n)$ classes be $p$-regular. There exist $n$ distinct (absolutely) irreducible characters $\chi_{1}, \chi_{2}, \ldots, \chi_{n}$ of $G$. Let 0 be the ring of all algebraic integers of $\Omega$ and let $p$ be a prime ideal of $\mathfrak{o}$ dividing $p$. If we denote by $0^{*}$ the ring of all $\mathfrak{p}$-integers of $\Omega$, then $\mathfrak{p}$ generates an ideal $\mathfrak{p}^{*}$ of $\mathfrak{p}^{*}$ and we have

$$
\Omega^{*}=\mathfrak{D}^{*} / \mathfrak{p}^{*} \cong \mathfrak{D} / \mathfrak{p}
$$

for the residue class field. The residue class map of $0^{*}$ onto $\Omega^{*}$ will be denoted by an asterisk; $\alpha \rightarrow \alpha^{*}$.

Let $\Gamma=\Gamma(G)$ be the modular group ring of $G$ over $\Omega^{*}$ and let

$$
Z=Z_{1} \oplus Z_{2} \oplus \cdots \oplus Z_{s}
$$

be the decomposition of the center $Z=Z(G)$ of $\Gamma$ into indecomposable ideals $Z_{\sigma}$. Then the ordinary irreducible characters $\chi_{i}$ and the modular irreducible characters $\varphi_{\kappa}$ of $G$ (for $p$ ) are distributed into $s$ blocks $B_{1}, B_{2}, \ldots, B_{s}$, each $\chi_{i}$ and $\varphi_{\kappa}$ belonging to exactly one block $B_{s}$. We determined in [6] explicitly the primitive orthogonal idempotents $\delta_{\sigma}$ of $Z$ corresponding to $B_{\sigma}$ in the following way. We set

$$
b_{\alpha}=\sum_{\chi_{i} \in B_{\sigma}} z_{i} \chi_{i}\left(a_{\alpha}^{-1}\right) / g \quad\left(a_{\alpha} \in K_{\alpha}\right)
$$

where $z_{i}=\chi_{i}(1)$. Let $U_{\kappa}$ be the indecomposable constituent of the regular representation of $G$ corresponding to the modular irreducible representation $F_{\kappa}$ and denote by $u_{\kappa}$ its degree. We see that $b_{\alpha}=\sum_{\varphi_{k} \in B_{\alpha}} u_{\kappa} \varphi_{\kappa}\left(a_{\alpha}^{-1}\right) / g \in 0^{*}$ for $p$-regular

Received July 13, 1965. 
classes $K_{\alpha}$ since $p^{a} \mid u_{\kappa}(\kappa=1,2, \ldots, m)$. On the other hand $b_{\alpha}=0$ for $m<\alpha$ $\leqq n$. Then we have

$$
\delta_{\sigma}=\sum_{\alpha=1}^{m} b_{\alpha}^{*} K_{\alpha}
$$

where the sum of the elements of $K_{\alpha}$ is also denoted by $K_{\alpha}$. In what follows we shall call $\delta_{\sigma}$ the block idempotents of $\Gamma$ associated with $B_{3}$ or simply the block idempotents of $B_{\sigma}$. Let $B_{\sigma}$ be a block of defect $d$ with defect group $D$. Then $b_{\alpha}^{*}=0$ if the defect group $D_{\alpha}$ of $K_{\alpha}$ is not contained in any conjugate of $D$ ([6], Theorem 4, see also [5]). Hence we obtain

$$
\delta_{\Omega}=\sum_{D_{\alpha} \subseteq D} b_{\alpha}^{*} K_{\alpha} \quad(1 \leqq \alpha \leqq m) .
$$

Here the notation $D_{\alpha} \subseteq D$ means that $D_{\alpha}$ is contained in some conjugate of $D$. In the special case where $p+g$, there exist $n$ modular irreducible characters of $G$. Further each $\chi_{i}$ forms a block $B_{\sigma}$ of its own. Hence

$$
\delta_{i}=\sum_{\alpha=1}^{n}\left(z_{i} \chi_{i}\left(a_{\alpha}^{-1}\right) / g\right)^{*} K_{\alpha} .
$$

We consider the fixed block $B=B$, of defect $d$ with defect group $D$. If we define $\nu(s)$ by $p^{\nu(s)} \| s$ for a rational integer $s$, then there exist characters $\chi_{k} \in B$ such that $\nu\left(z_{k}\right)=a-d$. We shall first prove that $l=\sum_{\alpha=1}^{m} \chi_{k}\left(a_{\alpha}^{-1}\right) \omega_{k}\left(K_{\alpha}\right)$ $\neq 0(\bmod p)$ where $\omega_{k}\left(K_{\alpha}\right)=g_{\alpha} \chi_{k}\left(a_{\varkappa}\right) / z_{k}$ and $g_{\alpha}$ denotes the number of elements of $K_{\alpha}$. The main purpose of this short note is to prove the following

Theorem 1. Let $\delta$ be the block idempotent of $B$ and let $\varepsilon=\sum_{\alpha=1}^{m} c_{\alpha}^{*} K_{\alpha}$ be an element of $Z$ where $c_{\alpha}=\%_{k}\left(a_{\alpha}^{-1}\right) / l$. Then $\delta-\varepsilon$ belongs to the radical of $Z$.

In the case where $p+g$ we see easily that this fact coincides with the formula (3) since $l=g / z_{k}$ for every $\chi_{k}$ and $\operatorname{rad} Z=0$.

Let $\chi_{i}$ be any character of $B$ and $\lambda_{i}$ be the height of $\chi_{i}$, that is, $\nu\left(z_{i}\right)=a$ $-d+\lambda_{i}\left(\lambda_{i} \geqq 0\right)$. Let $K_{\beta}$ be $p$-regular classes with defect group $D_{\beta}=D$. Then $\omega_{k}\left(K_{\beta}\right) \equiv \omega_{i}\left(K_{\beta}\right)(\bmod \mathfrak{p})$ and hence $g_{\beta} \chi_{k}\left(a_{\beta}\right) / z_{k} \equiv g_{\beta} \chi_{i}\left(a_{\beta}\right) / z_{i}(\bmod \mathfrak{p})$. Then it follows from $g_{3} / z_{k} \neq 0(\bmod \mathfrak{p})$ that

$$
\chi_{i}\left(a_{\beta}\right) \equiv\left(z_{i} / z_{k}\right) \chi_{k}\left(a_{\beta}\right) \quad\left(\bmod p^{\lambda i} \mathfrak{p}\right) .
$$

Since the modular irreducible characters of $B$ can be expressed by the ordinary irreducible characters of $B$ (restricted to $p$-regular elements) with integral 
coefficients, we have for $\hat{\varphi}_{\kappa} \in B$

$$
\varphi_{\kappa}=\sum_{x_{i} \in B} r_{\kappa i} \chi_{i}
$$

Hence, by (4)

$$
\varphi_{\kappa}\left(a_{\beta}\right) \equiv \sum_{\chi_{i} \in B}\left(r_{\kappa i} z_{i} / z_{k}\right) \chi_{k}\left(a_{\beta}\right) \quad(\bmod \mathfrak{p})
$$

and consequently

$$
\varphi_{\kappa}\left(a_{\beta}\right) \equiv\left(f_{\kappa} / z_{k}\right) \%_{k}\left(a_{\beta}\right) \quad(\bmod p)
$$

where $f_{\kappa}=\varphi_{\kappa}(1)$.

Lemma 1. Let $\chi_{k} \in B$ be the character of height 0 . Then $\sum_{\alpha=1}^{m} \chi_{k}\left(a_{\alpha}^{-1}\right) \omega_{k}\left(K_{\alpha}\right)$ \#0 $(\bmod \mathfrak{p})$.

Proof. It follows from (5) that

$$
\begin{aligned}
b_{\beta} & =\sum_{\eta_{K} \in B} u_{\kappa} \varphi_{\kappa}\left(a_{\beta}^{-1}\right) / g \\
& \equiv \sum_{\eta_{K} \in B_{B}}\left(u_{\kappa} f_{\kappa} / g z_{k}\right) \psi_{k}\left(a_{\beta}^{-1}\right) \quad(\bmod \mathfrak{p})
\end{aligned}
$$

and hence

$$
b_{\beta} \equiv\left(\sum_{i \in B} z_{i}^{2} / g z_{k}\right) \chi_{k}\left(a_{\beta}^{-1}\right) \quad(\bmod \mathfrak{p})
$$

for $p$-regular classes $K_{\beta}$ with defect group $D_{\beta}=D$. Since there exist $p$-regular classes $K_{\curlyvee}$ with defect group $D_{\curlyvee}=D$ such that $b_{\curlyvee} \neq 0(\bmod \mathfrak{p})$ and $\chi_{k}\left(\boldsymbol{a}_{\Upsilon}^{-1}\right) \neq 0$ $(\bmod \mathfrak{p})$, we obtain from $(6)$

$$
h=\sum_{x_{i} \in B} z_{i}^{2} / g z_{k} \neq 0 \quad(\bmod p) .
$$

It follows from (2) that

$$
\sum_{D \beta=D} b_{\beta} \omega_{k}\left(K_{\beta}\right) \equiv 1 \quad(\bmod \mathfrak{p})
$$

since $\omega_{k}\left(K_{\alpha}\right) \equiv 0(\bmod \mathfrak{p})$ for $p$-regular classes $K_{\alpha}$ with defect group $D_{\alpha}$ properly contained in some conjugate of $D$. Then we have by (6) and (7)

$$
h \sum_{D \beta D} \gamma_{k}\left(a_{\beta}^{-1}\right) \omega_{k}\left(K_{\beta}\right) \equiv 1 \quad(\bmod \mathfrak{p}) .
$$

Hence we see

$$
\sum_{D_{\beta}=D} \chi_{k}\left(a_{\beta}^{-1}\right) \omega_{k}\left(K_{\beta}\right) \neq 0 \quad(\bmod \mathfrak{p}) .
$$

If $\omega_{k}\left(K_{\alpha}\right) \neq 0(\bmod \mathfrak{p})$, then $D \subseteq D_{\alpha}$ and if $D$ is properly contained in some con- 
jugate of $D_{\alpha}$, then $\chi_{k}\left(a_{\alpha}\right) \equiv 0(\bmod p)$. Hence

$$
\sum_{\alpha=1}^{m} \chi\left(a_{\alpha}^{-1}\right) \omega_{k}\left(K_{\alpha}\right) \equiv \sum_{D \beta=D} \chi_{k}\left(a_{\beta}^{-1}\right) \omega_{k}\left(K_{\beta}\right) \quad(\bmod p)
$$

which proves the lemma.

We set $l=\sum_{\alpha=1}^{m} \chi_{k}\left(a_{\alpha}^{-1}\right) \omega_{k}\left(K_{\alpha}\right)$ and $c_{\alpha}=\chi_{k}\left(a_{\alpha}^{-1}\right) / l$ and consider the element $\xi=\sum_{\alpha=1}^{m} c_{\alpha} K_{\alpha}$ of the center of the ordinary group ring of $G$. Then

$$
\omega_{k}(\xi)=\sum_{\alpha=1}^{m} \chi\left(a_{\alpha}^{-1}\right) \omega_{k}\left(K_{\alpha}\right) / l=1
$$

and hence for any $\chi_{i} \in B$ we have $\omega_{i}(\xi) \equiv 1(\bmod \mathfrak{p})$. On the other hand, for any $\chi_{j} \notin B$

$$
\omega_{j}(\xi)=\sum_{\alpha=1}^{m} \chi_{k}\left(a_{\alpha}^{-1}\right) \omega_{j}\left(K_{\alpha}\right) / l=0
$$

because $\sum_{\alpha=1}^{m} g_{\sigma} \chi_{k}\left(a_{\alpha}^{-1}\right) \chi_{j}\left(a_{\alpha}\right)=0$. This implies that if we set $\varepsilon=\sum_{\alpha=1}^{m} c^{*} K_{\alpha}$, then $\delta$ $-\varepsilon \in \operatorname{rad} Z$. This completes the proof of Theorem 1 .

If $d_{\alpha}>d$ where $d_{\alpha}$ denotes the defect of $K_{\alpha}$, then $\chi_{k}\left(a_{\alpha}\right) \equiv 0(\bmod \mathfrak{p})$ and hence $c_{\alpha}^{*}=0$. Further if $d_{\alpha}=d$ and $D_{\alpha}$ is not conjugate to $D$, then $\omega\left(K_{\alpha}\right) \equiv 0$ $(\bmod p)$ and $\chi_{k}\left(a_{\alpha}\right) \equiv 0(\bmod p)$. Thus we have also $c_{\alpha}^{*}=0 . \quad$ It follows from (6), (7) and (8) that $b_{\beta}^{*}=c_{\beta}^{*}$ for all $p$-regular classes $K_{\beta}$ with defect group $D_{\beta}=D$.

Lemma 2. Let $Q$ be the normal p-subgroup of $G$. Then the block idempotent $\delta$ of $B$ with defect group $D$ is given by

$$
\delta=\sum_{Q \subseteq D \alpha \subseteq D} b_{\alpha}^{*} K_{\alpha} \quad(1 \leqq \alpha \leqq m) .
$$

Proof. We see that $b_{\alpha}^{*}=0$ for $p$-regular classes $K_{\alpha}$ such that $Q$ is not contained in $D_{\alpha}([6])$. This, combined with (2) proves the lemma.

Theorem 2. Let $B$ be the block of $G$ with normal defect group $D$. Then

$$
\varepsilon=\sum_{D \beta=D} c_{\beta}^{*} K_{\beta} \quad(1 \leqq \beta \leqq m)
$$

is the block idempotent of $B$ where $c_{\beta}=\chi_{k}\left(a_{\beta}^{-1}\right) / l$ and $l=\sum_{D_{\beta}=D} \chi_{k}\left(a_{\beta}^{-1}\right) \omega_{k}\left(K_{\beta}\right)$.

Proof. It follows from Lemma 2 that $\delta=\sum_{D_{\beta}=D} b_{\beta}^{*} K_{\beta}$. Then $\delta=\varepsilon$ since $b_{\beta}^{*}$ 
$=c_{\beta}^{*}$ for all $p$-regular classes $K_{\beta}$ with defect group $D_{\beta}=D$.

Now let $B_{1}$ be the principal block of $G$ which contains the principal character $\psi_{1}=1$ and let $\delta_{1}$ be its block idempotent. Obviously we may choose $\chi_{1}$ as the character $\chi_{k}$ in Theorem 1 . We then have $l=v$ where $v$ denotes the number of $p$-regular elements in $G$. If $Q$ is a $p$-Sylow subgroup of $G$, then $v \equiv u(\bmod p)$ where $u$ denotes the number of $p$-regular elements in the centralizer $C_{G}(Q)$. Hence

$$
\varepsilon_{1}=(1 / v)^{*} \sum_{\alpha=1}^{m} K_{\alpha}=(1 / \boldsymbol{u})^{*} \sum_{\alpha=1}^{m} K_{\alpha} .
$$

If $Q$ is normal in $G$, then we see by Theorem 2 that

$$
\varepsilon_{1}=(1 / u)^{*} \sum_{D \beta=Q} K_{\beta} \quad(1 \leqq \beta \leqq m)
$$

is the block idempotent $\delta_{1}$ of $B_{1}([7])$.

Some applications of our results will be presented elsewhere.

\section{REFERENCES}

[1] R. Brauer, Zur Darstellungstheorie der Gruppen endlicher Ordnung, I, Math. Zeits. 63 (1956), 406-444.

[2] R. Brauer, Zur Darstellungstheorie der Gruppen endlicher Ordnung, II, Math. Zeits. 72 (1959), 25-46.

[3] R. Brauer, Some applications of the theory of blocks of characters of finite groups, I, J. Alg. 1 (1964), 152-167.

[4] R. Brauer and W. Feit, On the number of irreducible characters of finite groups in a given block. Proc. Nat. Acad. Sci. U.S.A. 45 (1959), 361-365.

[5] C. W. Curtis and I. Reiner, Representation Theory of Finite Groups and Associative Algebras, Interscience, New York, London, 1962.

[6] M. Osima, Notes on blocks of group characters, Math. J. Okayama Univ. 4 (1955), 175-188.

[7] M. Osima, On a theorem of Brauer, Proc. Japan Acad. 40 (1964), 795-798.

\section{Osaka University}

